\title{
Performance Analysis of Translucent Space Division Multiplexing Based Elastic Optical Networks
}

\author{
Sridhar Iyer and Shree Prakash Singh
}

\begin{abstract}
The required upgradation of the network capacity of the single-mode fiber which is constrained by the non-linear Shannon's limit, and the capacity provisioning needed by the future diverse Internet traffic can be resolved by the adoption of the Space Division Multiplexing (SDM) based Elastic Optical Networks (EONs) (SDM-b-EONs). In the current work, we focus on the performance analysis of a SDM-b-EON in which translucent lightpaths are routed through the spectral superchannels over the spatial single-mode fiber(s) bundle(s) links. In regard to regeneration, we investigate three scenarios which differ in their regeneration variability level in addition to the adjustment of modulation formats according to transmission route characteristics. We conduct extensive simulations considering an online traffic case and two realistic network topologies with different numbers of (i) fibers in every link, and (ii) transceivers available within SDM-b-EON. The obtained results demonstrate that when regeneration is conducted with complete flexibility and simultaneously the modulation format conversion is also permitted at every SDM-b-EON node both, largest traffic volume amounts can be provisioned, and significant SDM-b-EON performance scaling can be obtained with a corresponding increase in the utilized fibers amount.
\end{abstract}

Keywords-Elastic optical networks, space division multiplexing, regeneration, resource allocation, network optimization.

\section{INTRODUCTION}

The continuously increasing diverse traffic and its related bandwidth demands are incrementally exhausting the available capacity of the optical networks. Recent studies have identified that the demands for the large capacity and the heterogeneous granularity in the next-generation optical network's traffic can be quenched by the adoption of the flexi-grid elastic optical networks (EONs) [1, 2]. Also, through a change in the subcarriers' amount and adaptation of the modulation format (MF), the EONs make it possible to (i) provision elastic bandwidth, and (ii) enable spectral super-channels. The aforementioned is accomplished by the deployment of the bandwidth flexible transponders and spectrum selective switches along a demand's path, hence ensuring the elasticity of the spectrum for the lightpaths in the EONs [3]. In the EONs, the main objective is to assign

Manuscript received December 8, 2018, revised January 16, 2019.

Sridhar Iyer is with Department of ECE, Jain College of Engineering, T S. Nagar Hunchanatti Cross-Machhe, Belagavi, Karnataka, India 590014. (e-mail: sridhariyer1983@gmail.com).

Shree Prakash Singh is with Division of ECE, Netaji Subhas Institute of Technology, Sector-3, Dwarka, New Delhi-110078. (e-mail: sps_nsit@yahoo.co.uk). resources by finding solutions to the Routing, Modulation Format, and Spectrum Assignment (RMFSA) problem, which has been proven to have NP-hard complexity [4].

However, it has been envisioned that even with the deployment of the flexi-grid Elastic Optical Networks (EONs), the capacity crunch will persist since, the EONs use only two multiplexing dimensions which in turn limit their throughput by the fiber capacity [5]. This crunch in capacity can be ameliorated with the adoption of space division multiplexing (SDM) technique in which, many spaces (fibers, cores or modes) are used in parallel thus provisioning an increase in the used spectral resources [6]. The major advantages provided by the SDM technique include flexible spectrum allocation, adaptive use of various MFs, and the super-channel transmission [7]. Further, using the SDM technique with the EON technology, as in the SDM based EONs (SDM-b-EONs), provides (i) immense flexibility in managing the spectrum and space, (ii) large capacity increase, and (iii) substantial savings in overall network cost [8]. Hence, with drastic traffic increase, space dimension expansion can be viewed as the only future-proof solution which will be able to provide the expected capacity.

Unlike the EONs, in which the major challenge is to solve the routing and spectrum allocation (RSA) problem [9], with the introduction of 'space', in a SDM-b-EON, the major challenge is to solve the routing, space, and spectrum allocation (RSSA) problem, whose solutions aim to optimally serve the demands over a path by appropriately allocating the spectrum and the space resources [10]. The RSSA problem transforms into routing, core, and spectrum allocation (RCSA) problem if multi-core fibers (MCFs) are used as the fiber solution [11]. Further, as an extension, the choice of the MF can also be included within the RSSA which then transforms it into the routing, space, modulation format and spectrum allocation (RSMFSA) problem [12].

In regard to the SDM-b-EONs, many fiber solutions exist such as, the single-mode fiber(s) $(\operatorname{SMF}(s))$ bundles, the MCFs, the multi-fibers (MFs), and the few-mode fibers (FMFs) [10]. With adoption of any of the aforementioned fiber solution, it becomes essential to counteract the degradations that occur due to the crosstalk (XT) which may degrade the optical signal's transmission reach (TR) to such an extent that it may be required to adopt a less efficient however, more robust MF. Therefore, to ensure that there occurs the transmission for long distances in the presence of $\mathrm{XT}$ as well and the various linear and non-linear physical 
layer impairments (PLIs), it may be required to deploy regenerators within the network. The use of regenerators within the network in turn may frequently permit the use of the MFs which are efficient hence resulting in much lesser utilization of the spectrum and the transceivers (TrSCrs) [13]. Specifically, in regard to the super-channels, if the length of the considered route is in excess of the TR of the utilized MF, then it is necessary to regenerate the superchannel. Among the many existing methods used for regeneration within the network, the back to back $(\mathrm{BtB})$ operation of the TrSCrs to regenerate the signals is the most popular technique [14]. The BtB technique, in which the TrSCrs interconnections occur at the client end, leads to the generation of translucent lightpaths in which every transparent segment of the route can utilize different MFs and may also be allotted a varied slot of frequency; however, it must be ensured that every segment of the route satisfies the spectrum-continuity constraint [13]. In regard to the EONs, the issue of assignment of the regenerators has been extensively investigated in many studies [4].

\section{RELATED WORKS}

Many existing studies based on the SDM-b-EONs have investigated various issues under the consideration of the various fiber solutions with different assumptions. The authors in [10] have assumed different sub-channels sizes to investigate the trade-off that exists between the spectralefficiency and the TR. In [11], as an initial step for protecting a SDM-b-EON, the authors have designed a strategy which is independent of failures in addition to protecting the routes. The authors have evaluated the proposed strategy for different traffic load values considering realistic topologies. The obtained results demonstrated that the proposed strategy is efficient in provisioning pre-configured protection, and the node degree of the considered network topology significantly affects the blocking and the evaluated length of the route. The authors in [12] have addressed the RSSA problem in a MCF enabled SDM-b-EON considering optical switches which implement the architecture on demand technology. For the performance analysis, the authors have considered the XT impact in the MCFs. In [15], the authors have investigated the performance of various switching techniques considering the wavelength selective switches amount as a parameter of cost. The authors in [16] have conducted the evaluation of the power consumed by a multi-input multi-output digital signal processor which is then utilized to minimize the XT in a strongly coupled MCF system. In [17], to provision the demands in a joint switching based MCF enabled SDM-bEON, the author has aimed at the cost minimization while assigning the spectral and the spatial resources. Initially, it is established that in specific cases; use of the complete core allotment (CCA) technique, which uses all the MCF cores to provision demands, leads to the spectrum underutilization following which; the author has introduced a novel core adapting allotment (CAA) technique which adapts the cores amounts. The author has investigated the possibility of using the CAA technique to provision the spatial super-channels simultaneously ensuring the same spectral requirements as needed when the CCA technique is used. The simulation results show that by using the CAA technique, indeed there is a possibility of the TrSCrs amount being minimized in effect resulting in the spatial super-channels method being an economically practical solution.

As mentioned earlier, the issue of assignment of the regeneration has been investigated in many EON studies [4]; however, to best of the authors' knowledge, the regeneration assignment issue in the SDM-b-EONs has not been analyzed in any existing study. In the current work, we consider the regeneration assignment issue in a SDM-b-EON in which the translucent lightpaths are routed through the spectral super-channels over spatial SMF bundle(s) links. We deliberate three different regeneration scenarios; wherein, every scenario differs in its regeneration variability level and the adjustment of the MFs as per the characteristics of the route which is chosen for transmission. We perform extensive simulations considering an online traffic case and two realistic network topologies with varying fiber amounts per link, and the availability of different $\mathrm{TrSCr}$ amounts within the network. We present the results in terms of Bandwidth Blocking Probability (BwBP), mean resource (i.e., spectrum and $\mathrm{TrSCrs}$ ) utilization, mean regeneration points amount, and distribution of the MFs.

The rest of the paper is structured as follows: Section III details the major assumptions on the considered network and the transmission model adopted in the study. In Section IV, we present and discuss the various obtained simulation results. Finally, Section V concludes the study.

\section{NETWORK AND TRANSMISSION MODEL}

In the current study, for the transmission, we adopt a similar model as in our previous study [13] in which, the optical signals are transmitted as the spectral super-channels with every super-channel being carried over the SMFs within the bundle. It must be noted that the optical carriers which form a super-channel use the same MF. The SDM-b-EON uses an elastic grid with a granularity of $12.5 \mathrm{GHz}$ and is also assumed to utilize the coherent TrSCrs which support reconfigurable bit-rates and various MFs as per properties of the optical link. The TrSCrs operate at a baud-rate which is fixed to a value of $28 \mathrm{Gbaud}$, and an optical carrier, whose transmission or reception is provisioned by a $\mathrm{TrSCr}$, utilizes three frequency slices which amounts to $37.5 \mathrm{GHz}$ of spectrum. Further, super-channels are separated by a guard band of one frequency slice which amounts to $12.5 \mathrm{GHz}$.

In view of the TRs of the considered MFs, we adopt the values from the study in [8]. Hence, the bit-rates supported by a $\mathrm{TrSCr}$ is in accordance with the spectral-efficiency of a specific MF, as shown in Table I. Also, if it occurs that the bit-rate of a demand is in excess of the utmost capacity supported by a $\mathrm{TrSCr}$ which uses a specific MF, the demand is permitted to be provisioned by multiple optical carriers which are transmitted within one super-channel, and which occupy an adequate adjacent slices numbers. Hence, for a case in which the quadrature phase shift keying (QPSK) format is implemented to support a $100 \mathrm{Gbps}$ demand, but the demand bit-rate is $400 \mathrm{Gbps}$, a super-channel will use 4 TrSCrs, and will hence need 12 frequency slices. 
TABLE I

TRANSMISSION REACH VALUES FOR THE VARIOUS MODULATION FORMATS, AND THE BIT-RATE OF EACH TRANSCEIVER.

\begin{tabular}{ccc}
\hline Modulation Format & $\begin{array}{c}\text { Transmission } \\
\text { Reach }(\mathrm{km})\end{array}$ & $\begin{array}{c}\text { Bit-rate } \\
(\mathrm{Gbps})\end{array}$ \\
\hline binary phase shift keying (BPSK) & 6607 & 50 \\
quadrature phase shift keying (QPSK) & 3311 & 100 \\
16-quadrature amplitude modulation (QAM) & 832 & 150 \\
64-quadrature amplitude modulation (QAM) & 209 & 200 \\
\hline \hline
\end{tabular}

In the current study, we consider the following three regeneration scenarios:

- Least Regeneration (LR): In this scenario, the aim is to reduce the regeneration amount by ensuring that a lightpath comprises of the least transparent segments amount [4]. LR allows for the conversion of the spectrum; however, the MF conversion is not permitted and hence, the MF which has been selected for the longest segment of the route is also employed for all the other segments.

- Complete Flexibility (CF): In this scenario, flexibility for the regeneration in the complete sense is assumed i.e., if needed, the MF conversion is permitted at every network node [18]. The main aim is the utilization of the MF with higher spectral-efficiency for transparent segments which have shorter length with an additional possibility to employ a varied MF over the different segments of the lightpath.

- CF without MF Conversion (CFwoMFC): We introduce this scenario in which, as in the $\mathrm{CF}$ case, the regeneration can be conducted at any node; however, as in the LR case, the MF conversion is not permitted in addition to no requirement on the reduction of the regeneration amount.

It must be noted that in order to provision a demand, the use of every regeneration scenario splits the transmission route into multiple transparent segments of the route, and for every such segment, the 'best' permissible MF is chosen. Further, every regeneration scenario is different in its requirement for the TrSCrs and the spectrum amounts in addition to the network location where these TrSCrs and spectrum resources are required. Therefore, as per the TrSCrs and the spectrum resources which are available, the 'best' configuration is chosen with an aim to enhance the online demand serving flexibility.

\section{Simulation Results And Discussions}

For the performance evaluations, we consider two realistic network topologies: the Telefónica (TID), and the GEANT; the details of which can be found in [13]. It is assumed that every SDM-b-EON link is a SMFs bundle and every fiber provides a spectrum which is equal to $4 \mathrm{THz}$ that amounts to 320 frequency slices of $12.5 \mathrm{GHz}$ each. With an aim to analyze the nature of performance scaling of the SDM-bEON considering the different regeneration scenarios, we vary the SMFs amount within a bundle in the range of 2-10 with a granularity of 2 . Every $\mathrm{TrSCr}$ supports a specific bit- rate which depends on the MF used, which is shown in Table I. As mentioned earlier, in the case when the demanded bitrate is larger than the largest capacity of one $\mathrm{TrSCr}$ which uses a certain MF, the provisioning of the demand occurs through multiple optical carriers, each of which (i) use the same MF, (ii) is transmitted within the same super-channel, and (iii) is assigned enough neighboring frequency slices amount separated by a guard band of $12.5 \mathrm{GHz}$. The simulations are conducted using the CPLEX optimizer [19] and the MATLAB software.

We use the $k$-shortest path algorithm with $k=5$ shortest paths with the inclusion of the routing and the regeneration configurations for generating the permissible candidate lightpaths. It is also assumed that the TrSCrs are already deployed in the network before the routing process is executed. We use a uniform distribution to allocate the TrSCrs which ensures that each network node is equipped with a similar amount of the coherent TrSCrs which function at a set baud rate.

The demands consist of stochastically generated end nodes, and the bit-rates spanning 50 Gbps - 1 Tbps having a granularity of $50 \mathrm{Gbps}$. The arrival of demands follows a Poisson process with a mean rate of arrival rate equal to $\lambda$ demands every unit of time. For every demand, the holding time is generated in accordance with a negative exponential distribution which has a $1 / \mu$ mean value. Hence, the load of traffic which is offered equals $\lambda / \mu$ normalized units of traffic (NUTs). Further, between the source and the destination nodes, the demands are distributed uniformly which implies that there is an equal probability of every node being chosen either as a source node or a destination node. Lastly, for every load of traffic value which is investigated, a single set with 100,000 demands is simulated; however, the initial 10,000 demands are not investigated for allowing the network to achieve a steady state.

Lastly, to compare the performance of the considered BtB regeneration scenarios, we consider the traffic which is accepted for a 1 percent BwBP threshold value [7] i.e. any specific case is executed for the different NUT values in view of determining the largest traffic which is acceptable within the network such that the BwBP does not exceed a value of 1 percent. In regard to the TrSCrs amount, we vary the amount in the range of 2000-20,000 with a granularity of 2000. For the comparison, we also show the results in terms of the mean spectrum and the TrSCrs utilization, mean regeneration points amount, and distribution of MFs.

In Fig. 1 and 2, for the GEANT and the TID topologies, we show the variation of the accepted traffic by every fiber corresponding to a 1 percent $\mathrm{BwBP}$ threshold with the already deployed TrSCrs amount considering all the regeneration scenarios. It can be observed from the figures that for all the regeneration scenarios in both the considered topologies, as the TrSCrs amount increases, initially, the traffic amount which is accepted by every fiber also increases. Further, on reaching a certain point, the traffic accepted by every fiber starts to stabilize with the stabilizing point differing for every regeneration scenario. Also, this point of stabilization is observed to be dependent on the fibers amount and the regeneration scenario. 


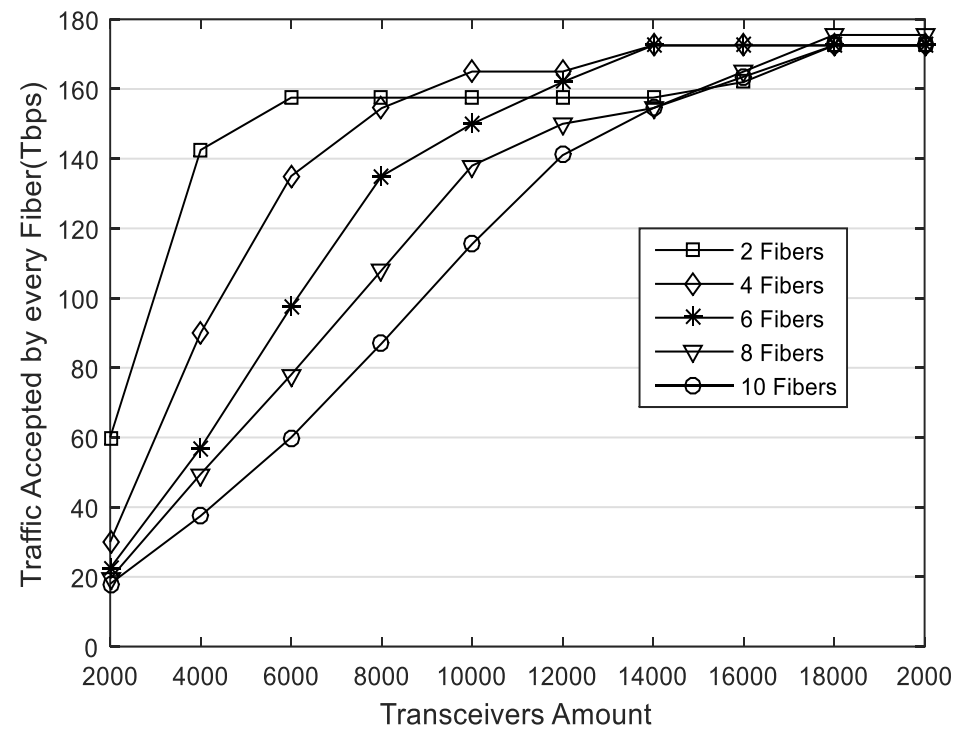

(a)

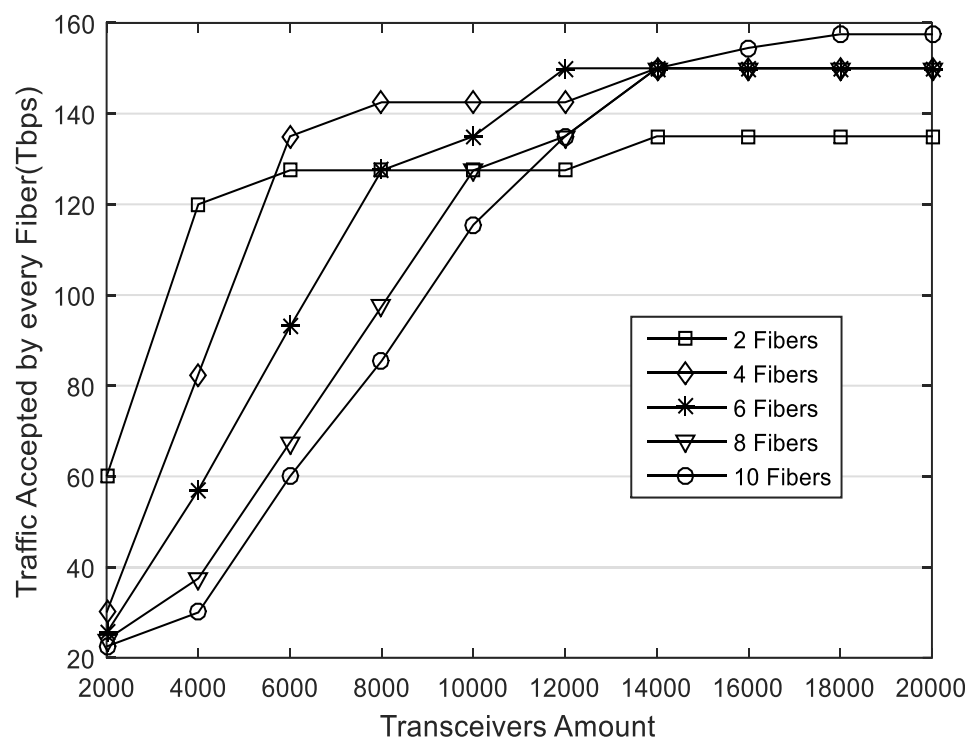

(b)

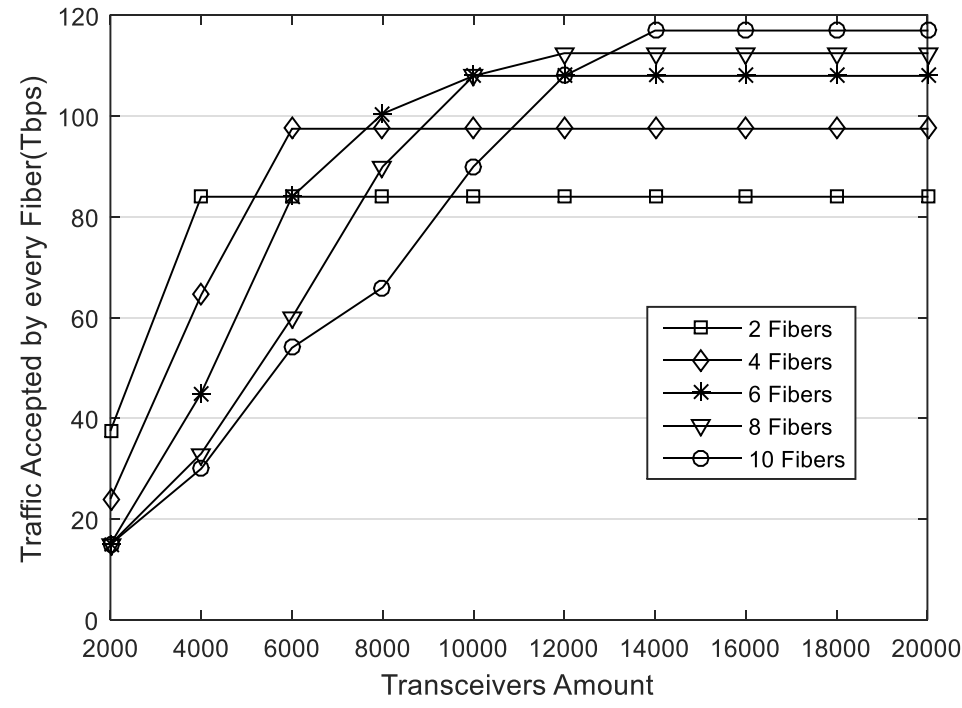

(c)

Fig. 1. Variation of the accepted traffic by every fiber corresponding to 1 percent BwBP threshold with the transceivers amount for the GEANT topology considering the: (a) CF regeneration scenario, (b) CFwoMFC regeneration scenario, (c) LR regeneration scenario. 


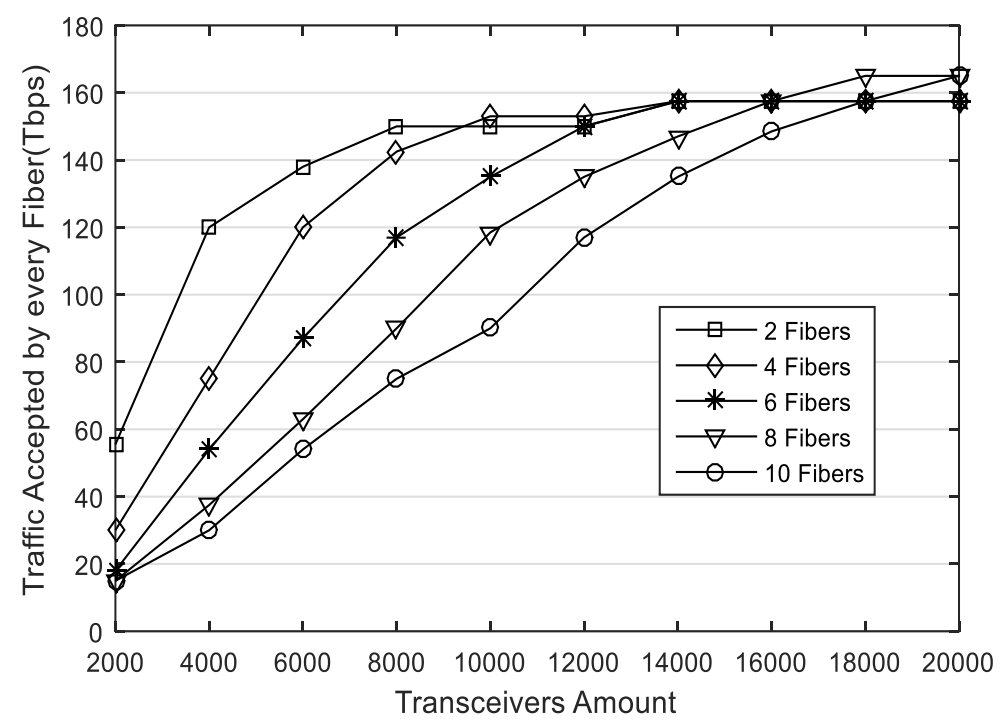

(a)

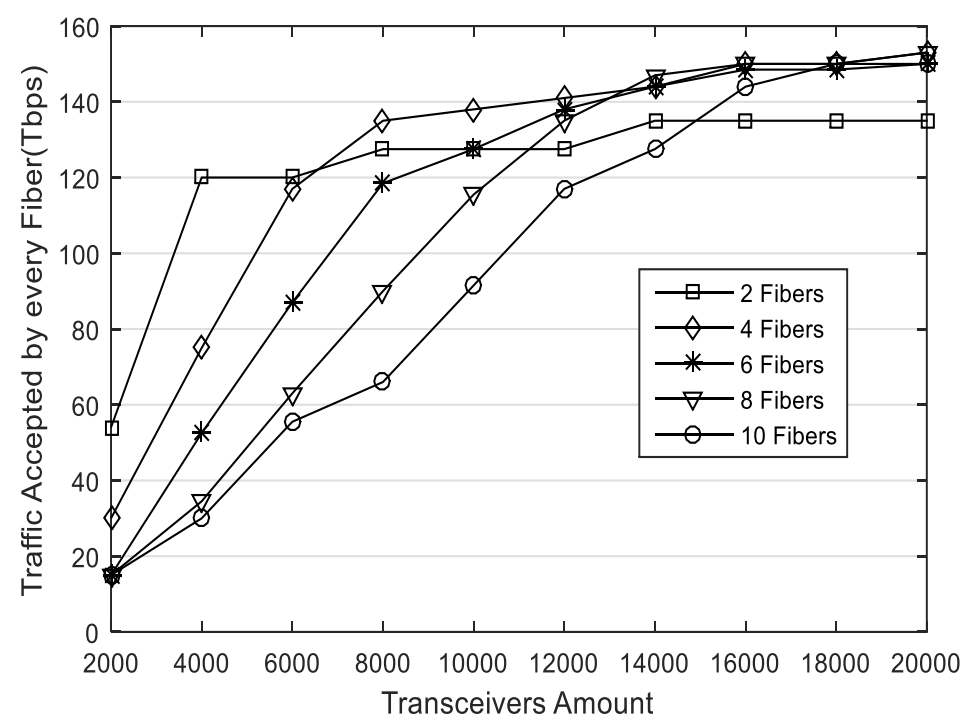

(b)

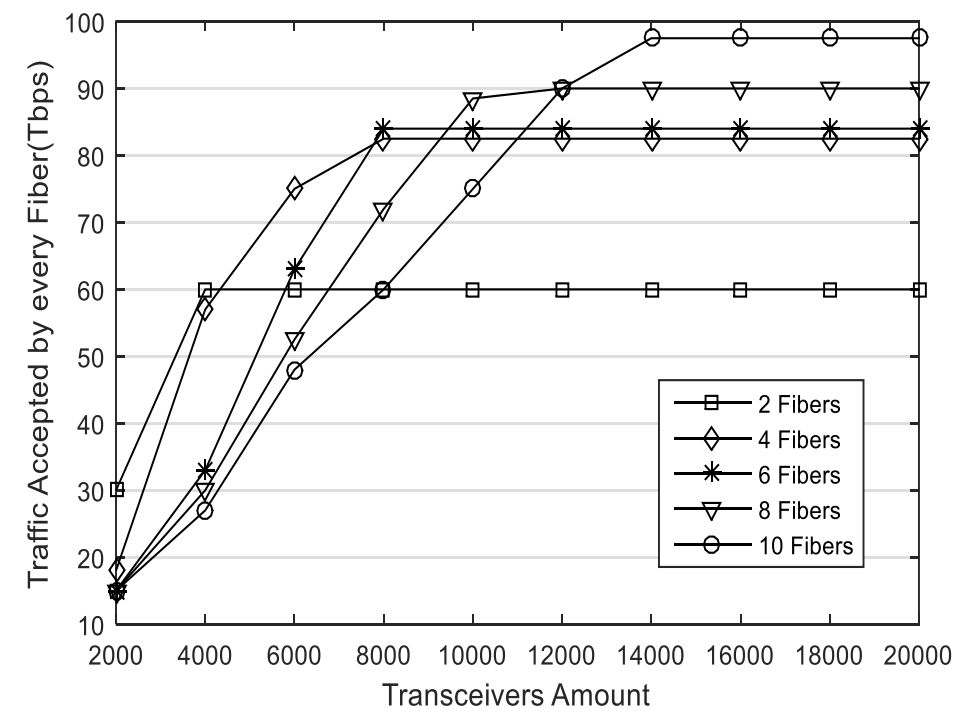

(c)

Fig. 2. Variation of the accepted traffic by every fiber corresponding to 1 percent BwBP threshold with the transceivers amount for the TID topology considering the: (a) CF regeneration scenario, (b) CFwoMFC regeneration scenario, (c) LR regeneration scenario. 
It can also be observed from the figures that considering the GEANT topology, the CF regeneration scenario is able to provision the highest amount of accepted traffic by every fiber which is approximately 178 Tbps. For the same topology, the CFwoMFC scenario provides the next best results as it is able to accept approximately $160 \mathrm{Tbps}$ of traffic per fiber whereas, the LR scenario show the worst performance by accepting approximately $118 \mathrm{Tbps}$ traffic by every fiber. Hence, it can be inferred that the $\mathrm{CF}$ regeneration scenario outperforms other two regeneration scenarios as it is able to best utilize the resources (spectrum, TrSCrs, and fibers) which are available. Therefore, if the same resource(s) amount is available then, compared to the other regeneration scenarios, the $\mathrm{CF}$ regeneration scenario will result in the largest income for any network operator by provisioning the largest traffic amount.

The results in Fig. 1 and 2 also highlight the efficient SDM-b-EON performance scaling provided by the $\mathrm{CF}$ regeneration scenario which implies the variation in the SDM-b-EON performance with varied SMFs amount within the bundle. It can be observed from the figures that in regard to the CFwoMFC scenario, when there are adequate TrSCrs amount within the network then, the accepted traffic by every fiber demonstrates the same value convergence.

The aforementioned implies that the SDM-b-EON proportionally uses the extra fibers (i.e., space resource). However, in comparison, the $\mathrm{CF}$ regeneration scenario is able to permit much higher allocation of the traffic (approximately 18 percent). The LR regeneration scenario demonstrates a large difference in the results obtained for varied fibers amount.

Overall, it can be concluded from Fig. 1 and 2 that the CF regeneration scenario is able to outperform the other regeneration scenarios specifically for the higher TrSCrs amount and lesser fibers amount within the bundle. Hence, we obtain the results that follow considering the $\mathrm{CF}$ regeneration scenario and the GEANT topology. It must be noted though that due to limited space in the article we do not present the results corresponding to the TID topology which also generated similar trends of the results.

Next, we demonstrate in Fig. 3, the mean spectrum and the TrSCrs amount utilization, and the mean regeneration points amount for the $\mathrm{CF}$ regeneration scenario. We find the results for 1 percent $\mathrm{BwBP}$ threshold in the GEANT topology considering varied fiber amount. It can be observed from the figure that when the $\mathrm{CF}$ regeneration scenario is used in the SDM-b-EON, then the resource usage scales efficiently. However, for the various fibers amount, the performance is observed to be strongly dependent on the available TrSCrs amount.

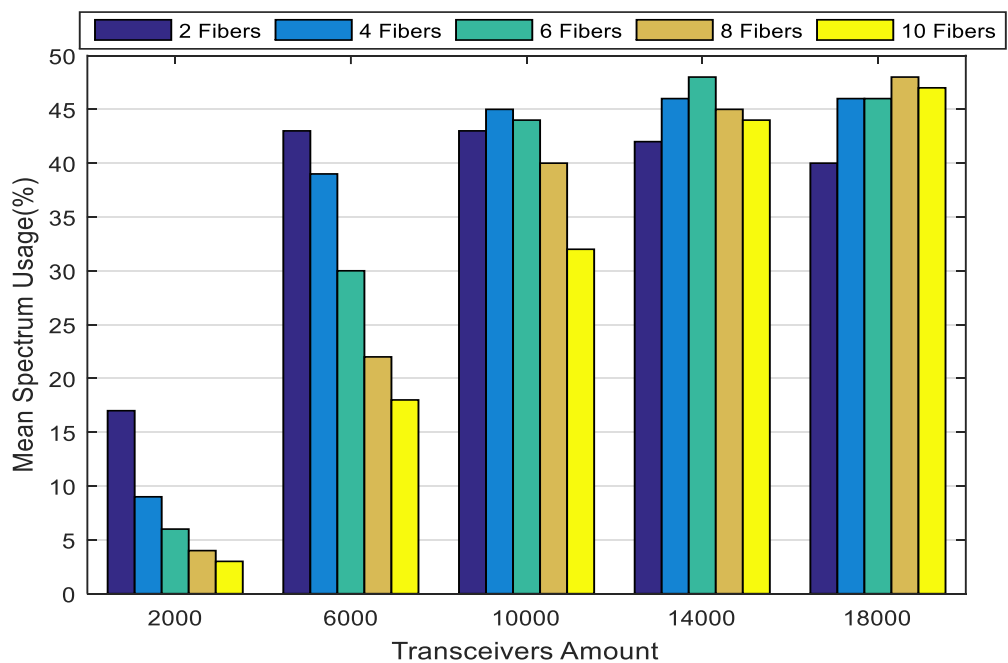

(a)

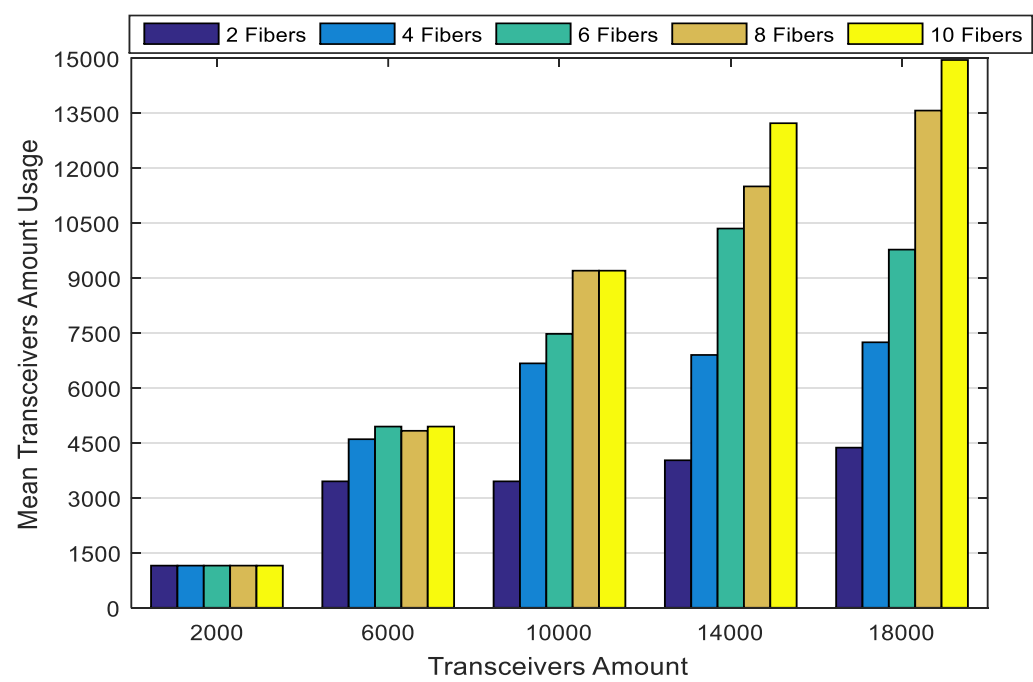

(b) 


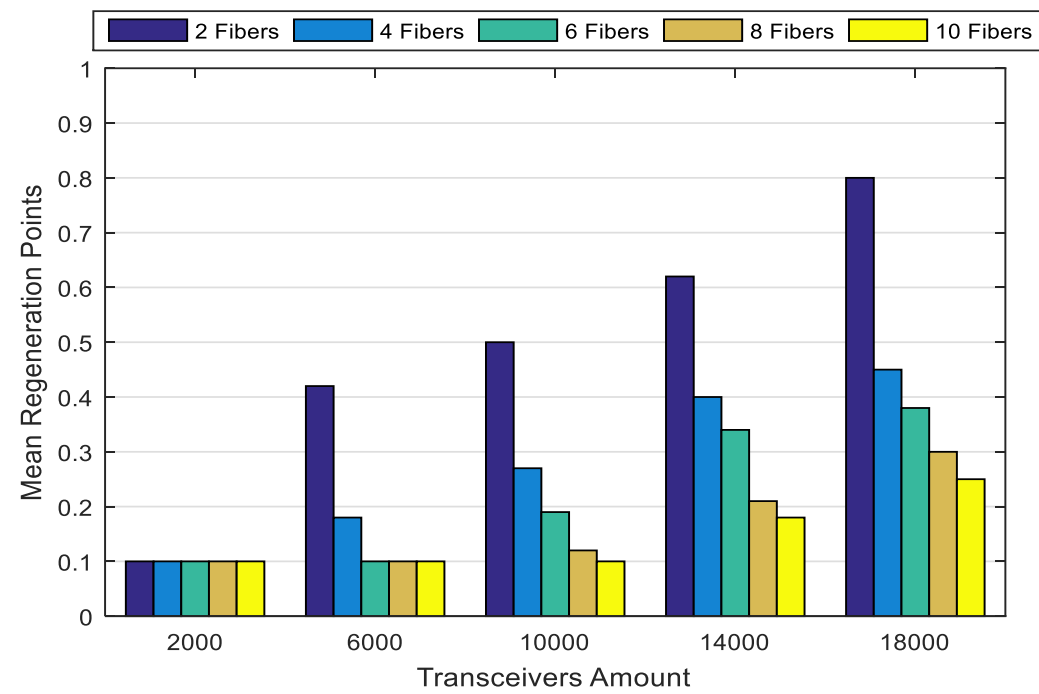

(c)

Fig. 3. Performance of the CF regeneration scenario observed for 1 percent BwBP threshold in the GEANT topology in terms of: (a) mean spectrum utilization, (b) mean transceivers amount used, (c) mean regeneration points amount.

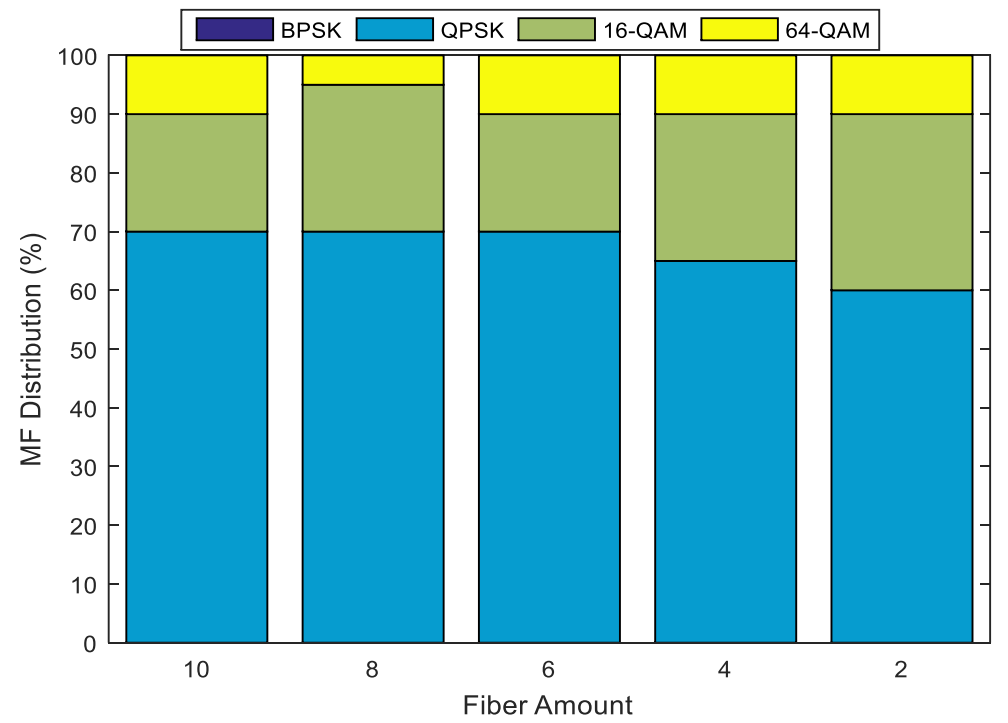

(a)

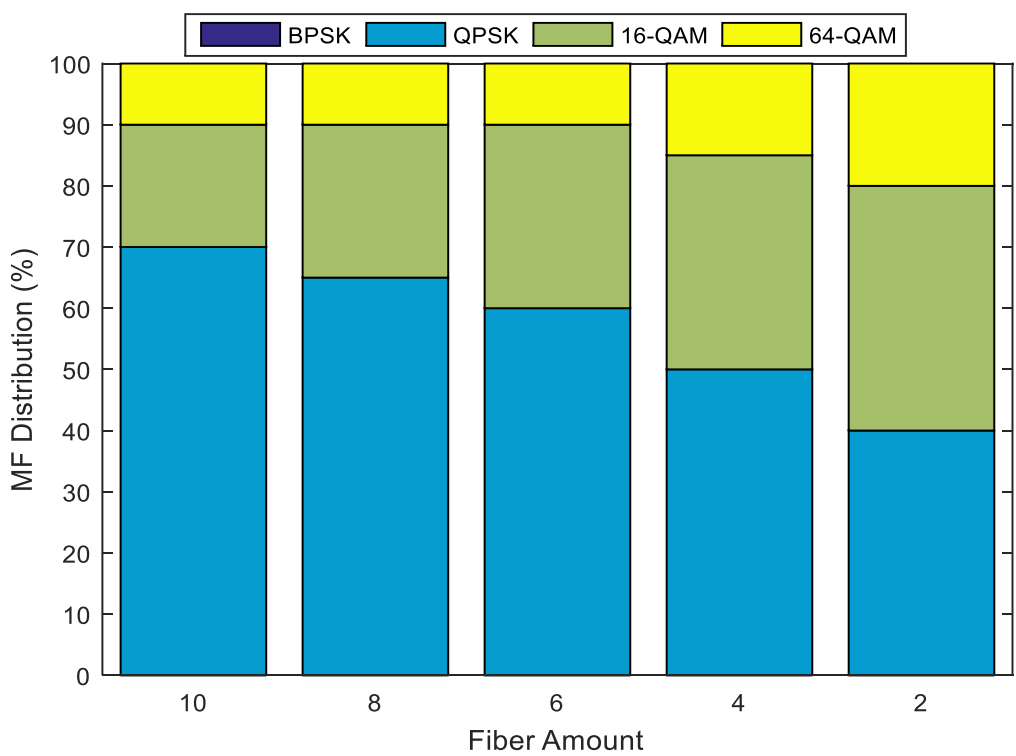

(b) 


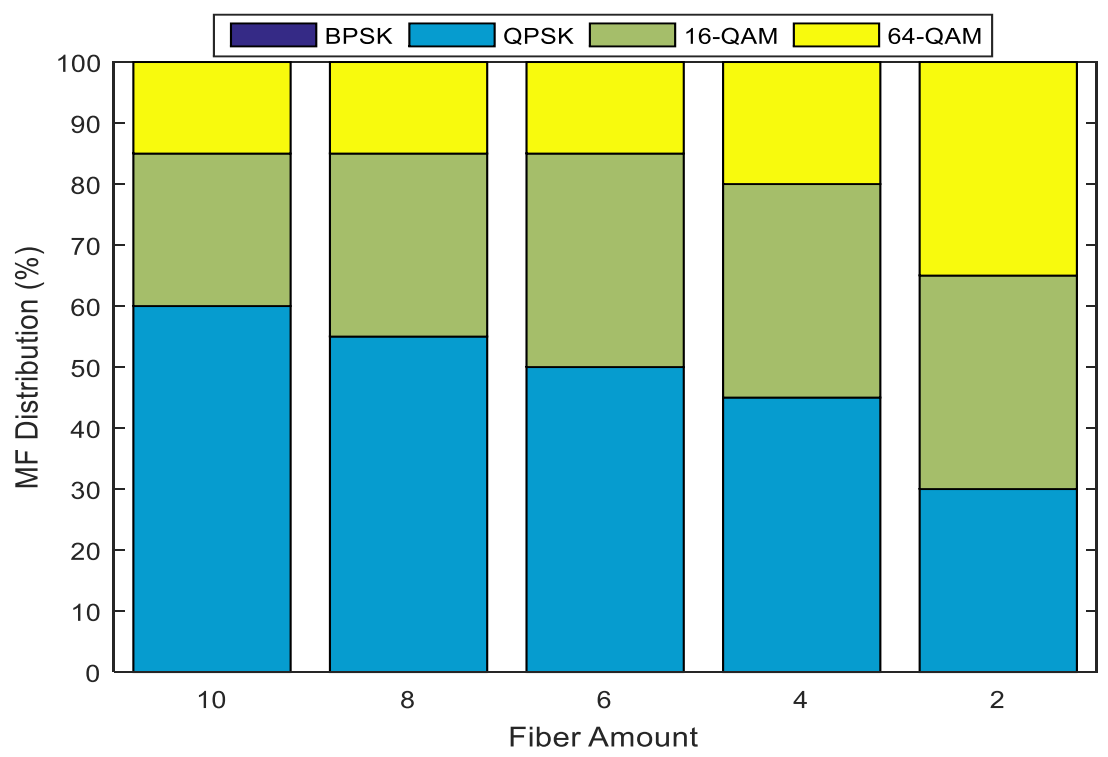

(c)

Fig. 4. Variation of the percentage MFs utilization with fibers amount corresponding to 1 percent BwBP threshold in the CF regeneration scenario and GEANT topology considering the transceiver amounts as (a) 2000, (b) 10000 and, (c) 18000.

Lastly, we demonstrate the variation of percentage MFs utilization with the fibers amount (see Fig. 4). Again, to obtain the results we consider the 1 percent BwBP threshold and the $\mathrm{CF}$ regeneration scenario in the GEANT topology in which the TrSCrs amounts vary as 2000, 10000, and 18000 . It can be observed from the figure that when the fibers amount is less then, more often, the MFs with higher spectral-efficiency (i.e., 16-QAM, QPSK) are utilized. The aforementioned is a result of the fact that for a fixed TrSCrs amount when the fibers amount is less then, the accepted traffic amount is also less and as a result larger TrSCrs amount can be utilized for the regeneration. This in turn leads to the route segment which is much shorter and larger utilization of the MFs with higher spectral-efficiency. Furthermore, as the available TrSCrs amount is increased, the MFs with even higher spectral-efficiency, such as 64QAM and 16-QAM, can be used (see Fig. 4 (c)).

\section{CONCLUSION}

In the current work, we focused on the performance analysis of a translucent SDM-b-EON enabled by the SMFs bundles. For enabling the long distance transmission and the simultaneous use of spectrally efficient modulation formats, we assumed the use of the back to back method in which the transceivers are used for regeneration. We then deliberated three different regeneration scenarios namely: Least Regeneration (LR), Complete Flexibility (CF), and $\mathrm{CF}$ without MF Conversion (CFwoMFC). The aforementioned regeneration scenarios differ in the manner in which they vary the regeneration and are also able to adjust the modulation formats as per transmission route characteristics.

We conducted extensive simulations considering an online traffic case and two realistic network topologies. In the simulations, we assumed different fiber amounts in every link and the availability of different transceiver amounts in the network. The obtained results have demonstrated that the $\mathrm{CF}$ regeneration scenario is able to outperform the other two considered regeneration scenarios. In its part, the $\mathrm{CF}$ regeneration scenario provisions the 'best' utilization of the available fibers, spectrum and transceivers which implies that with the given resources, the $\mathrm{CF}$ regeneration scenario can provision the highest traffic amount which in turn provides significant benefits to a network operator. Furthermore, we also showed that the network performance is least affected by the available fibers amount in every network link. Finally, we analyzed in detail the utilization of the resources and the MFs with a variation in the available resources. As a scope for future research, we will conduct a similar analysis considering other fiber solutions.

\section{REFERENCES}

[1] O. Gerstel, M. Jinno, A. Lord, and S.J. Yoo, "Elastic Optica Networking: A New Dawn for the Optical Layer", IEEE Commun. Mag., vol. 50, no. 2, pp. S12-S20, 2012.

[2] M. Jinno, "Elastic optical networking: roles and benefits in beyond 100-Gb/s era", IEEE J. Lightw. Technol., vol. 35, no. 5, pp. 11161124, 2017.

[3] A. Napoli, M. Bohn, D. Rafique, A. Stavdas, N. Sambo, L. Potì, M. Nölle, J.K. Fischer, E. Riccardi, A. Pagano, A. Di Giglio, M.S Moreolo, J.M. Fabrega, E. Hugues-Salas, G. Zervas, D. Simeonidou, P. Layec, A. D’Errico, T. Rahman, and J.P.F-Palacios Giménez, "Next Generation Elastic Optical Networks: The Vision of the European Research Project IDEALIST", IEEE Commun. Mag., vol. 53, no. 2, pp. 152-162, 2015 .

[4] B. Chatterjee, N. Sarma, and E. Oki, "Routing and spectrum allocation in elastic optical networks: A tutorial", IEEE Commun. Surv. \& Tutorials., vol. 17, no. 3, pp. 1776-1800, 2015.

[5] D. Klonidis, F. Cugini, O. Gerstel, M. Jinno, V. Lopez, E. Palkopoulou, M. Sekiya, D. Siracusa, G. Thouénon, and C. Betouleet, Spectrally and Spatially Flexible Optical Network Planning and Operations, IEEE Commun. Mag., vol. 53, no. 2, pp. 69-78, 2015.

[6] G. M. Saridis, D. Alexandropoulos, G. Zervas, and D. Simeonidou, "Survey and evaluation of space division multiplexing: From technologies to optical networks," IEEE Commun. Surv. \& Tutorials. vol. 17, no. 4, pp. 2136-2156, 2015.

[7] M. Klinkowski, P. Lechowicz, and K. Walkowiak, "Survey of resource allocation schemes and algorithms in spectrally-spatially flexible optical networking," Opt. Switch. Netw., vol. 27, no. 1, pp. 58-78, 2018.

[8] R. Rumipamba-Zambrano, J. Perelló, J. M. Gené, and S. Spadaro, 'Cost-effective spatial super-channel allocation in Flex-Grid/MCF 
optical core networks', Opt. Switch. Netw., vol. 27, pp. 93-101, 2018.

[9] S. Iyer, and S.P. Singh, "Offline Scheduling Schemes to Transfer Voluminous Deadline Complying Data in Elastic Optical Networks", in Proc. IEEE $24^{\text {th }}$ National Conference on Communications (NCC2018), IIT-H, India, 2018, pp. 1-6.

[10] P. S. Khodashenas, J. Manuel Rivas-Moscoso, D. Siracusa, F. Pederzolli, B. Shariati, D. Klonidis, E. Salvadori, and I. Tomkos, Comparison of Spectral and Spatial Superchannel Allocation Schemes for SDM Networks, IEEE J. Lightw. Technol., vol. 34, no. 11, pp. 2710-2716, 2017.

[11] S. Iyer, and S.P. Singh, "A Novel Protection Strategy for Elastic Optical Networks Based on Space Division Multiplexing", in Proc. IEEE $12^{\text {th }}$ International Conference on Signal Processing and Communication (SPCOM-2018), IISC-Bengaluru, India, 2018, pp.15.

[12] A. Muhammad, G. Zervas, and R. Forchheimer, Resource Allocation for Space-Division Multiplexing: Optical White Box Versus Optical Black Box Networking, IEEE J. Lightw. Technol., vol.33, no.23, pp. 4928-4941, 2015.

[13] S. Iyer, "Solutions to Large Cases of RSA with Transceiver and Regeneration Allocation (RSAwTCRA) Problem in Elastic Optical Networks", Photon. Netw. Commun., 2017. DOI: 10.1007/s11107017-0726-8

[14] A. Fallahpour, H. Beyranvand, S. A. Nezamalhosseini, and J. Salehi, "Energy efficient routing and spectrum assignment with regenerator placement in elastic optical networks," IEEE J. Lightw. Technol., vol. 32, no. 10, pp. 2019-2027, 2014

[15] B. Shariati, D. Klonidis, D. Siracusa, F. Pederzolli, J. M. RivasMoscoso, L. Velasco, and I. Tomkos, "Impact of traffic profile on the performance of spatial superchannel switching in SDM networks," in Proc. IEEE European Conf. on Optical Communication (ECOC), 2016, pp. 1-3.

[16] N. P. Diamantopoulos, B. Shariati, and I. Tomkos, "On the power consumption of MIMO processing and its impact on the performance of SDM networks," in Proc. OSA Optical Fiber Communications Conf. and Exhibition (OFC), 2017.

[17] S. Iyer, "On the Cost Minimization in Space Division Multiplexing Based Elastic Optical Networks", J. Opt. Commun., De Gruyter 2018. DOI: https://doi.org/10.1515/joc-2018-0127.

[18] K. Christodoulopoulos, P. Soumplis, and E. Varvarigos "Planning flexible optical networks under physical layer constraints," IEEE/OSA J. Opt. Commun. Netw., vol. 5, no. 11, pp. 1296-1312, 2013.

[19] IBM. ILOG CPLEX optimizer, 2017. Available: http://www.ibm.com.

Sridhar Iyer received the B.E. degree in Electronics and Telecommunications Engineering from Mumbai University, India in 2005, M.S degree in Electrical and Communication Engineering from New Mexico State University, U.S.A in 2008, and the Ph.D. degree from Delhi University, India in 2017. He worked as an Assistant Professor in the Department of ECE at NIIT University, and Christ University, India between 2012 - 2016. He received the young scientist award from the Department of Science and Technology, Government of India in the year 2013. Currently he is an Associate Professor in the Department of ECE, Jain College of Engineering, India. His research interests include the architectural, algorithmic, and performance aspects of the optical networks, with current emphasis on efficient design and resource optimization in the Space Division Multiplexing enabled flexi-grid Elastic optical networks. Dr. Iyer has published over 50 peer-reviewed articles in the aforementioned areas.

Shree Prakash Singh received his B.E. degree in Electronics and Communication Engineering from MMM Engineering College, Gorakhpur, India, in 1990 and M.Tech. degree from REC, Kurukshetra, India, in 1994. $\mathrm{He}$ holds a Doctoral Degree in Optical Networks from the Indian Institute of Technology, Delhi (IITD), India, in 2007. During 1994-2000, he worked as Lecturer in the Department of Electronics and Computer Engineering at REC Kurukshetra. In October 2000, he joined as Lecturer in the Department of Electronics, Netaji Subhas Institute of Technology (formally known as Delhi Institute of Technology New Delhi). Presently he is Professor at the Division of Electronics and Communication Engineering, Netaji Subhas Institute of Technology, New Delhi. His current area of research is Optical Networks, Optical wireless communication. 